\title{
Avaliação de habilidades sociais em pacientes com anomalias da diferenciação sexual
}

\author{
Evaluation of social skills in patients with disorders of sex development
}

Ana Paula de Andrade Sardinha', Eleonora Arnaud Pereira Ferreira²

Resumo Anomalias da diferenciação sexual (ADS) correspondem a um conjunto de malformações congênitas na genitália. É frequente indivíduos com ADS apresentarem dificuldades em interações sociais; entretanto, há poucos estudos sobre este tema. O termo Habilidades Sociais (HS) se refere a repertórios comportamentais que se caracterizam por interações sociais satisfatórias. Objetivo: Neste trabalho, fez-se a avaliação de comportamentos relacionados a HS em indivíduos com ADS atendidos em ambulatório especializado. Método: Participaram nove adultos com idade entre 21 a 35 anos. Utilizou-se: Protocolo para análise de prontuário, Roteiro de entrevista semi-estruturada e o Inventário de Habilidades Sociais (IHS). Comparou-se a média e o desvio padrão dos participantes da amostra, classificada segundo a identidade de gênero, com a média e o desvio padrão da amostra normativa masculina e feminina do IHS. Resultados: Os resultados indicaram déficit de HS em todos os participantes nos cinco fatores do IHS. Considerações finais: Discute-se sobre a importância de se intervir precocemente com indivíduos com ADS visando à prevenção de déficits em HS além do tratamento médico convencional.

Descritores: habilidades sociais; anomalia da diferenciação sexual; inventário de habilidades sociais; identidade de gênero.

Summary Disorders of sex development (SDS) correspond to a set of congenital malformations in the genitalia. It is common for individuals with SDS to present difficulties in social interactions; however, there are few studies on this topic. The term Social Skills (SS) refers to behavioral repertoires characterized by satisfactory social interactions. Purpose: In this study, the SS-related behaviors were evaluated in individuals with SDS attended at a specialized outpatient clinic. Methods: Nine adults aged between 21 and 35 participated in the study. We used the Protocol for medical record analysis, semi-structured Interview Script and Social Skills Inventory (SSI). The mean and standard deviation of all the participants, categorized according to gender identity, were compared with the mean and standard deviation of the male and female normative sample of SSI. Results: The results indicated a deficit of SS in all participants in the five SSI factors. Conclusion: We discussed the importance of early intervention on individuals with SDS for the prevention of SS deficits in addition to conventional medical treatment.

Keywords: social skills; disorder of sex development; social skills inventory; gender identity.

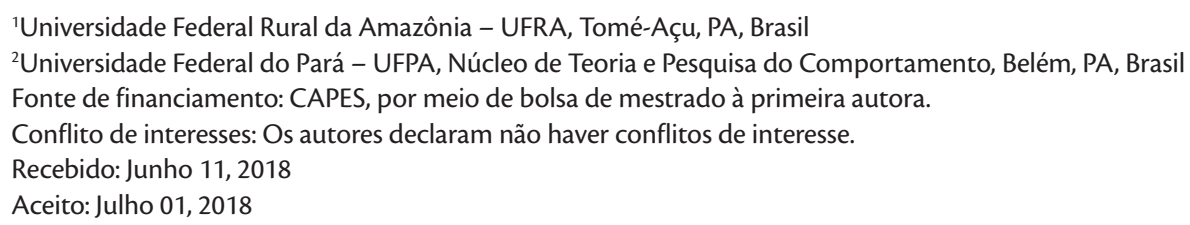

Trabalho realizado na Universidade Federal do Pará -UFPA, Hospital Universitário Bettina Ferro de Souza, Belém, PA, Brasil. 


\section{Introdução}

As anomalias da diferenciação sexual (ADS) são malformações congênitas que resultam desde ambiguidade genital clássica, visíveis ao nascimento, até infertilidade em adultos ${ }^{1-3}$. Trata-se de um desenvolvimento atípico ou incongruente do sexo genético, gonadal ou anatômico ${ }^{4}$, cuja prevalência é de 1:3000 a 1:5000 nascimentos ${ }^{5,6}$. É recomendável que a identificação de casos de ADS ocorra no período neonatal, momento em que uma investigação cuidadosa facilitaria o diagnóstico precoce para então iniciar as intervenções e o tratamento, prevenindo-se problemas emocionais e dificuldades de relacionamento social ${ }^{7}$. Entretanto, o mais comum ainda tem sido o diagnóstico ocorrer tardiamente, tornando mais desafiador o trabalho dos profissionais da equipe de saúde.

Devido à malformação na genitália e às características sexuais secundárias condizerem ou não com o sexo de criação e com a identidade de gênero, indivíduos com ADS podem se tornar vulneráveis a situações constrangedoras em seu convívio social ${ }^{6}$. Estudos sobre ADS já foram publicados, sendo a maioria na área médica com enfoque no tratamento ${ }^{8}$. Poucos investigaram questões emocionais relacionadas a estas anomalias, com destaque para habilidades facilitadoras de boas relações interpessoais nesses indivíduos ${ }^{9-13}$. Tais habilidades, intituladas habilidades sociais (HS), incluem não só a verbalização como também a expressão facial, a postura, o contato visual, gestos, cuidados com a aparência física, dentre outros ${ }^{14}$. Refere-se à expressão, pelo indivíduo, de atitudes, sentimentos, opiniões, desejos, respeitando a si próprio e aos outros, propiciando, em geral, resoluções dos problemas imediatos e diminuição da probabilidade de problemas futuros ${ }^{15}$. Entende-se que se trata de um problema de saúde, pois gera impacto psicológico importante em pacientes acometidos por ADS. Dessa forma, foi realizado um estudo com o objetivo de avaliar comportamentos correspondentes a HS em indivíduos adultos com ADS, preenchendo lacuna na área.

\section{Método}

O estudo se caracteriza como descritivo com delineamento transversal. Participaram pacientes com diagnóstico de ADS atendidos no serviço público de saúde de um hospital universitário (HU), e que concordaram em assinar o termo de consentimento livre e esclarecido (Projeto aprovado sob o Protocolo No. 79/08). Os critérios de inclusão foram: ter idade superior a 18 anos, com diagnóstico confirmado de ADS, e estar em tratamento há mais de seis meses no serviço de endocrinologia. Foram excluídos os que apresentassem déficit cognitivo que comprometesse seu entendimento a respeito dos objetivos da pesquisa e os que estivessem fazendo uso de medicamentos prescritos por serviço de psiquiatria.

A coleta de dados aconteceu em salas de atendimento ambulatorial do HU, e iniciou com a análise dos prontuários dos pacientes encaminhados ao Serviço de Psicologia pelos profissionais do ambulatório de Endocrinologia, com o objetivo de identificar os critérios de inclusão e de exclusão estabelecidos para a composição da amostra. Após a análise, os participantes pré-selecionados foram abordados em sala de espera e convidados a participar do estudo mediante leitura e assinatura do termo de consentimento. Em seguida, os participantes foram submetidos, individualmente, à aplicação do Inventário de Habilidades Sociais (IHS) ${ }^{16}$ e do Roteiro de entrevista semi-estruturada, nessa sequência. Durante a aplicação do Roteiro de entrevista, foi utilizado um gravador de áudio para o registro das respostas dos participantes. Após a aplicação dos instrumentos, foi agendada uma entrevista para devolução dos resultados obtidos com o IHS, em dia e horário acordados com cada participante. As entrevistas duraram em média 40 minutos. Na entrevista devolutiva, aqueles que apresentaram resultados indicativos de déficits em HS foram encaminhados ao serviço de Psicologia do HU para acompanhamento.

Os dados obtidos por meio dos prontuários e das entrevistas foram analisados e categorizados de acordo com o tema. A análise do IHS seguiu as instruções padronizadas, iniciando-se com a obtenção dos escores de cada participante considerando-se o escore total e os escores fatoriais (Fator 1- Habilidades de enfrentamento com risco; Fator 2- Habilidades de auto-afirmação na expressão de afeto positivo; Fator 3- Habilidades de conversação e desenvoltura social; Fator 4- Habilidades de auto-exposição a desconhecidos ou a situações novas; e Fator 5- Habilidades de autocontrole da agressividade em situações aversivas); em seguida, efetuou-se a comparação entre a média e o desvio padrão dos participantes da amostra masculina e da amostra feminina desse estudo com a média e o desvio padrão da amostra normativa masculina e feminina ${ }^{16}$. Foram incluídos na amostra masculina, os cinco participantes que declararam ter identidade de gênero masculina, enquanto na amostra feminina, as quatro participantes com identidade de gênero 
feminina. Para avaliar a significância da diferença entre as médias das habilidades de cada fator obtidas pelos participantes com as médias obtidas pela amostra normativa ${ }^{16}$ realizou-se o teste $t$ para diferença entre média de duas amostras independentes ${ }^{17}$.

\section{Resultados}

A amostra ficou composta por nove participantes com idade entre 21 a 35 anos. Três possuíam cariótipo 46XX e tanto o gênero de criação quanto a identidade de gênero eram femininos. Um participante com cariótipo 46XY possuía gênero de criação masculino, mas identidade de gênero feminina, assumindo o gênero feminino após o início do tratamento no ambulatório do HU. Quanto aos cinco participantes com identidade de gênero masculina, em todos houve correspondência entre o cariótipo e o gênero de criação. Quanto ao grau de escolaridade, apenas três participantes haviam completado o Ensino Médio. Entre os demais, quatro permaneciam estudando e dois haviam abandonado os estudos. Quanto ao local de residência, a maioria $(n=6)$ tinha domicílio no interior do Estado. Em termos de constituição familiar, apenas um participante residia com outra família que não a de origem.

A maioria $(n=5)$ dos participantes apresentou diagnóstico de deficiência na síntese de hormônio, seguido de hiperplasia adrenal congênita $(n=2)$ e hipogonadismo hipergonadotrófico $(n=2)$. Todos foram diagnosticados somente quando estavam na puberdade ou no início da idade adulta. Quatro participantes relataram que não faziam uso regular do medicamento indicado, alegando dificuldades financeiras para aquisição do remédio, ou mesmo pela baixa adesão às orientações do tratamento.

As queixas principais estavam relacionadas ao descontentamento com a aparência física ( $n=5)$, dúvidas em relação à orientação sexual $(n=2)$ e dúvidas quanto ao tratamento médico indicado $(n=2)$.

Quanto às queixas secundárias, todos os participantes apresentaram ao longo do atendimento dificuldades em HS, incluindo problemas de relacionamento com a mãe $(n=3)$, dificuldades no estabelecimento de amizades $(n=3)$, dificuldades no relacionamento sexual $(n=4)$, problemas quanto a atividades profissionais $(n=2)$. Foram consideradas como queixas principais aquelas que os participantes relatavam no primeiro atendimento com o serviço de psicologia e que também eram as descritas pelos médicos no encaminhamento do paciente a esse serviço. Como secundárias, as queixas relatadas ao longo dos atendimentos realizados no serviço de psicologia.

Dentre os participantes que estavam frequentando a escola na época da coleta de dados $(n=4)$, todos relataram dificuldades acadêmicas, predominando aquelas relacionadas ao entendimento do conteúdo ministrado durante as aulas. Também houve relato de dificuldade de relacionamento com colegas $(n=1)$ e de isolamento social no ambiente escolar $(n=1)$. Destaca-se que a maioria dos participantes $(n=6$ de 9$)$ abandonou as atividades escolares em algum momento de sua história de vida. Além disso, todos os participantes relataram ter passado por alguma situação de constrangimento social na escola devido à ADS. Tais constrangimentos, de acordo com os participantes, justificavam a evasão escolar, assim como a dificuldade em ingressar no mercado de trabalho.

Os resultados obtidos com a aplicação do IHS estão apresentados na Tabela 1.

Verificou-se que a média do escore total entre os participantes com identidade de gênero masculina foi de $85,6 \pm 39,43$ pontos, estando, portanto, abaixo da média da amostra normativa masculina $(96,00 \pm 13,6)$, sugerindo indicação para treino de HS. Entre as participantes com identidade de gênero feminina, obteve-se $90,5 \pm 41,57$ pontos no escore total, sugerindo também valores abaixo da média da amostra normativa feminina $(92,00 \pm 15,4)$, contudo podendo ainda ser considerado como um bom repertório social total ${ }^{16}$. O valor de $\mathrm{p}(\mathrm{T}<=\mathrm{t}$ ) bi-caudal obtido no escore total do IHS foi maior que 0,025 tanto para os participantes com identidade de gênero masculina quanto para os com identidade de gênero feminina; logo, a hipótese nula é aceita. Ou seja, a diferença entre as médias dos participantes e as da amostra normativa quanto ao escore total não se mostrou significativa, independentemente do gênero. No entanto, o valor de $\mathrm{p}(\mathrm{T}<=\mathrm{t})$ bi-caudal obtido em cada um dos cinco fatores do IHS foi menor que 0,025 Logo, pode-se aceitar a alternativa de que as médias são diferentes ao nível de significância de 5\%, ou seja, a diferença entre as médias dos participantes e as da amostra normativa mostrou-se significativa tanto para aqueles com identidade de gênero masculina quanto os com identidade de gênero feminina em todos os cinco fatores. Destaca-se os resultados obtidos no Fator 5 (Autocontrole da agressividade em situações aversivas), indicando necessidade de treino em HS para os participantes nesse quesito. 
Tabela 1. Comparação entre a média e o desvio padrão dos participantes com a média e o desvio padrão da amostra normativa quanto ao gênero e aos escores total e fatoriais em percentis obtidos no IHS e teste $t$ para diferença entre duas médias considerando variâncias desiguais

\begin{tabular}{|c|c|c|c|c|c|c|}
\hline \multirow{3}{*}{ Escores } & \multicolumn{3}{|c|}{ Gênero Masculino } & \multicolumn{3}{|c|}{ Gênero Feminino } \\
\hline & Participantes & \multicolumn{2}{|c|}{ Amostra $^{a}$} & Participantes & \multicolumn{2}{|c|}{ Amostra $^{a}$} \\
\hline & Média (DP) & \multicolumn{2}{|c|}{ Média (DP) $P$} & Média (DP) & \multicolumn{2}{|c|}{ Média (DP) $P$} \\
\hline Total & $85,6(39,43)$ & $96,00(13,6)$ & 0,5875 & $90,5(41,57)$ & $92,0(15,4)$ & 0,9471 \\
\hline Fator 1 & $7,42(3,15)$ & $10,35(2,7)$ & 1,95419E-05 & $9,468(3,95)$ & $8,4(3,2)$ & 0,000486005 \\
\hline Fator 2 & $8,16(3,26)$ & $8,79(1,7)$ & 1,61298E-05 & $9,14(3,9)$ & $9,62(1,7)$ & 2,55721E-05 \\
\hline Fator 3 & $6,45(3,05)$ & $6,72(1,4)$ & 7,61103E-05 & $6,36(3,64)$ & $6,59(1,35)$ & 0,000356383 \\
\hline Fator 4 & $2,08(3,21)$ & $3,3(1,22)$ & 0,000457123 & $2,71(3,8)$ & $3,32(1,28)$ & 0,002353883 \\
\hline Fator 5 & $0,732(3,45)$ & $1,24(0,8)$ & 0,001299633 & $0,185(4,24)$ & $0,89(0,8)$ & 0,015541126 \\
\hline
\end{tabular}

Nota: Fator 1- Enfrentamento com risco; Fator 2- Auto-afirmação na expressão de afeto positivo; Fator 3- Conversação e desenvoltura social; Fator 4- Auto-exposição a desconhecidos ou a situações novas; Fator 5- Autocontrole da agressividade em situações aversivas.

Fonte: Protocolo de pesquisa e a Amostra normativa ${ }^{16}$.

\section{Discussão}

Os resultados obtidos com a caracterização da amostra confirmam o atraso com que indivíduos com ADS são diagnosticados e iniciam o tratamento e acompanhamento especializado, como apontado pela literatura nacional ${ }^{12,18-20}$. O mesmo ocorreu em relação ao descontentamento com a aparência física (citado como a queixa principal), provavelmente devido a não correspondência entre a identidade de gênero e as características sexuais secundárias decorrentes do diagnóstico de ADS, pode estar associado ao histórico de dificuldades de relacionamento interpessoal descrito pelos participantes em entrevista ${ }^{20,21}$.

Os resultados obtidos com o IHS apontaram déficits em HS em todos os participantes da amostra em cada um dos cinco fatores analisados. Os déficits indicados para o Fator 1 (Enfrentamento com risco) sugerem que os participantes, independentemente da identidade de gênero, tinham dificuldade em lidar com situações que demandassem a defesa de seus direitos. A defesa dos próprios direitos está atrelada ao conceito de assertividade que, apesar de ser considerado como um comportamento altamente competente e habilidoso, também é visto como um comportamento interpessoal de risco a partir do momento em que possibilita a perda de reforçadores pela exposição do indivíduo à punição pelo interlocutor, ocasionando comportamentos de rejeição ou de oposição ${ }^{22}$. Os déficits no Fator 2 (Auto-afirmação na expressão de afeto positivo) apontam dificuldades para lidar com demandas de expressão de sentimentos positivos e de afirmação da autoestima ${ }^{16}$. Déficits no Fator 3(Conversação e desenvoltura social) sugerem desconhecimento de normas acerca de relacionamento interpessoal no cotidiano ou baixo desempenho em habilidades correspondentes a este fator ${ }^{16}$. Déficits no Fator 4 (Auto-exposição a desconhecidos ou a situações novas) incluem dificuldades em abordar pessoas desconhecidas, correspondendo à esquiva de expor-se a situações aversivas, como reações agressivas ou constrangedoras por parte dos interlocutores.

Dentre os fatores que compõem o IHS, tanto os participantes com identidade de gênero masculina quanto os com identidade de gênero feminina obtiveram resultados com indicativo de treino no Fator 5 (Autocontrole da agressividade em situações aversivas). Déficits neste fator correspondem à dificuldade de reagir a estimulações aversivas do interlocutor, perdendo-se o controle da raiva e da agressividade. Correspondem à dificuldade em lidar com críticas ou com chacotas, refletindo características de impulsividade com demanda para treino em HS. O ideal seria que, nestes casos, a raiva fosse expressa de forma considerada socialmente competente, no que se refere ao controle dos sentimentos negativos, com demonstração da consciência de seus direitos ${ }^{16}$. Comparando-se os resultados do Fator 5 com os relatos dos participantes durante as entrevistas, aprender a lidar com chacotas seria uma demanda importante para treino de HS com estes participantes.

Observou-se que houve maior dispersão nos resultados obtidos no escore total do IHS com os participantes deste estudo ao se comparar com a dispersão observada na amostra normativa. Este resultado sugere a 
necessidade de se considerar os casos individualmente, identificando-se na amostra aqueles participantes que apresentassem maiores déficits em HS e que poderiam se beneficiar com atendimento especializado, assim como identificar participantes que, a despeito do diagnóstico de ADS tardio, conseguiram desenvolver repertório social geral habilidoso. Ao avaliar-se o repertório geral de HS dos participantes, deve-se ainda frisar o fato de que, em uma primeira avaliação, os informantes podem superestimar seus desempenhos, autoanalisando-se bastante positivamente ${ }^{23}$, o que poderia ser verificado em estudos com medidas repetidas.

Os resultados sugerem que os participantes deste estudo poderiam se beneficiar com um treinamento de HS como parte do protocolo de tratamento, uma vez que se trata de uma técnica que visa à superação e/ou redução dos déficits por eles apresentados ${ }^{24}$. Destaca-se, também, a importância de se intervir precocemente com indivíduos com ADS, identificando-os ainda em idade pediátrica conforme sugere a literatura ${ }^{25}$, visando à prevenção de déficits em HS além do tratamento médico convencional.

\section{Considerações finais}

Este estudo permitiu a identificação de déficits em HS em adultos com ADS, apontando dificuldades enfrentadas por eles no convívio social, as quais podem estar vinculadas às alterações fenotípicas que estas anomalias ocasionam. É de grande relevância social diante das peculiaridades dos casos de ADS, apontando que além de aspectos médicos, aspectos sociais e psicológicos devem ser considerados no tratamento. Ao término dessas considerações, destacam-se: (1) a relevância em se investir no tratamento de indivíduos com ADS, o que envolveria não somente o tratamento médico, mas também o psicoterapêutico; e (2) os poucos estudos voltados para a área de saúde quanto ao treino em HS, uma vez que a maioria das pesquisas publicadas sobre essa temática aborda situações em ambiente escolar. Por isso, espera-se que este estudo contribua para enriquecer a literatura de ADS e HS, objetivando favorecer a uma melhor qualidade de vida de pessoas acometidas por essa anomalia. Concluindo, sugere-se a realização de mais estudos na área ampliando-se a investigação em relação ao número de participantes no serviço público de saúde.

\section{Referências}

1. Bosinski HA. Psychosexual aspects of intersex syndromes. Urologe. 2006;45(8):981-91. http://dx.doi.org/10.1007/s00120-006-1097-4. PMid:16835789.

2. Damiani D, Steinmetz L. Critérios diagnósticos. In: Maciel-Guerra AT, Guerra-Júnior G. editors. Menino ou menina? Distúrbios da diferenciação do sexo. Rio de Janeiro: Rubio; 2010. p.75-78.

3. Spinola-Castro AM. Aspectos históricos e éticos dos distúrbios da diferenciação do sexo. In: Maciel-Guerra AT, Guerra-Júnior G. (Eds.), Menino ou menina? Distúrbios da diferenciação do sexo. Rio de Janeiro, RJ: Rubio; 2010. p. 455-477.

4. Damiani D, Guerra-Junior G. As novas definições e classificações dos estados intersexuais: o que o Consenso de Chicago contribui para o estado da arte? Arq Bras Endocrinol. Met. 2007;51(6):1013-1017.

5. Andrade JGR, Martins RRS, Caldas D, Brasil J, Meiriño ALA, Jung MP. Perfil clínico de 62 casos de distúrbios da diferenciação sexual. Rev Paul Pediatr. 2008;26(4):321-8. http://dx.doi.org/10.1590/S0103-05822008000400003.

6. Hemesath TP. Anomalias da diferenciação sexual: representações parentais sobre a constituição da identidade de gênero. Psicol Reflex Crit. 2013;26(3):583-90. http://dx.doi.org/10.1590/S0102-79722013000300018.

7. Guimarães A, Barboza HH. Designação sexual em crianças intersexo: uma breve análise dos casos de "genitália ambígua". Cad. Saúde Pública. 2014;30(10):2177-2186.

8. Aguiar, MFM; Dias, DB; Caldato, M; Ferreira, EAP; Bernardes, JGB; Fonseca, RC. Hermafroditismo verdadeiro: relato de caso. Rev Para Med; 2008;22(4).71-74.

9. Andrade JGR, Martins RRS, Caldas D, Brasil J, Meiriño ALA, Jung MP. Perfil clínico de 62 casos de distúrbios da diferenciação sexual. Rev Paul Pediatr. 2008;26(4):321-8. http://dx.doi.org/10.1590/S0103-05822008000400003.

10. Damiani D, Guedes DR, Damiani D, Dichtchekenian V, Coelho JR No, Guerra AM, et al. Homem XX: relato de três casos na faixa etária pediátrica. Arq Bras Endocrinol Met. 2005;49(1):79-82.

11. Machado PS. “Quimeras” da ciência: a perspectiva de profissionais da saúde em casos de intersexo. Rev Bras Cienc Soc. 2005;20(59):67-80.

12. Maciel-Guerra A, Guerra G Jr. Ambigüidade genital: classificação. In: Maciel-Guerra A, Guerra G Jr, organizadores. Menino ou menina? os distúrbios da diferenciação do sexo. São Paulo: Manole; 2002. p. 31-36. 
13. Houk CP, Levitsky LL. Evaluation of the infant with atypical genitalia (disorder of sex development). UpToDate [Internet]. 2016. [citado em 2018 mai 28]. Disponível em: https://www.uptodate.com/contents/evaluation-of-the-infantwith -atypical-genitalia-disorder-of-sex-development?source=search_result\&search=genitalia\%20ambigua\&selectedTitle=1 56

14. Del Prette ZA. Psicologia das habilidades sociais: terapia e educação. Petrópolis: Vozes; 2011.

15. Caballo VE. Manual de avaliação e treinamento das habilidades sociais. São Paulo: Santos Editora; 2012.

16. Del Prette ZA, Del Prette A. Inventário de habilidades sociais (IGS-Del-Prette): manual de aplicação, apuração e interpretação. São Paulo: Casa do Psicólogo; 2001.

17. Martins GA, Domingues O. Estatística geral e aplicada: revisada e ampliada. São Paulo: Atlas Editora; 2014.

18. Benchaya I. Caracterização de pacientes portadores de anomalia da diferenciação sexual atendidos em um hospital universitário [dissertação]. Belém (PA): Universidade Federal do Pará. Manuscrito não publicado; 2008.

19. Brunhara FCR, Petean EBL. Hiperplasia Congênita de supra-renal: a compreensão do diagnóstico e implicações para a auto-imagem. Medicina. 2003;(36):45-53.

20. Sardinha APA. Habilidades sociais em portadores de anomalia da diferenciação sexual [tese]. Belém (PA): Universidade Federal do Pará; 2010.

21. Bittencourt Z, Ceschini M. Diagnóstico das ambiguidades genitais: avaliação social. In: Maciel-Guerra A, Guerra G Jr, organizadores. Menino ou menina? Os distúrbios da diferenciação do sexo. São Paulo: Manole; 2002. p. 191-201.

22. Delamater RJ, Mc Namara JR. The social impact of assertiveness: research findings and clinical implications. Behav Modif. 1986;10(2):139-58. http://dx.doi.org/10.1177/01454455860102001. PMid:3729911.

23. Del Prette A, Del Prette ZAP. Psicologia das relações interpessoais: vivências para o trabalho em grupo. Petrópolis: Vozes; 2007.

24. Bolsoni-Silva, AT. Habilidades sociais: breve análise da teoria e da prática à luz da análise do comportamento. Rev. Int. Psicol. $2002 ; 6(2): 233-242$.

25. Santos M, Araújo T. A clínica da intersexualidade e seus desafios para os profissionais de saúde. Psicol. Cienc. Prof. 2003;23(3):26-33. http:// dx.doi.org/10.1590/S1414-98932003000300005.

\section{Autor correspondente}

Ana Paula de Andrade Sardinha

Avenida Almirante Barroso (complemento), Alameda Irmã Consolata, 121, Marco

CEP 66095-230, Belém, PA, Brasil

Tel.: (91) 3236-1485; (91) 992422913

E-mail: ana.sardinha@ufra.edu.br; aprena@ig.com.br

\section{Informação sobre os autores}

APAS doutora em psicologia pelo Núcleo de Teoria e Pesquisa do Comportamento da Universidade Federal do Pará (NTPC/UFPA); docente da Universidade Federal Rural da Amazônia (UFRA); EAPF doutora em psicologia pela Universidade de Brasília (UNB); professora voluntária na Universidade Federal do Pará (UFPA), sendo orientadora de mestrado e doutorado no Programa de Pós-Graduação em Teoria e Pesquisa do Comportamento.

\section{Contribuição dos autores}

Ambas as autoras foram responsáveis pela elaboração do projeto, coleta e análise dos dados, revisão da literatura e redação final do artigo. Este estudo faz parte da dissertação de mestrado da Autora 1, sob orientação da Autora 2. 